\title{
Off Mass Shell Effects in Hadron Electric Dipole Moments
}

\author{
M.B. Hecht, B.H.J. McKellar \\ School of Physics, University of Melbourne, Parkville VIC 3052, Australia
}

(November 6, 2018)

\begin{abstract}
We note that off the quark mass shell the operators $\left(p_{i}+p_{f}\right)_{\mu} \gamma_{5}$ and $i \sigma_{\mu \nu}\left(p_{i}-\right.$ $\left.p_{f}\right)^{\nu} \gamma_{5}$, both of which reduce to $-\vec{\sigma} \cdot \vec{E}$ in the non-relativistic limit, are no longer identical. In this paper we explore the effects of this difference in the contribution of these quark electric moments to hadronic electric moments.
\end{abstract}




\section{INTRODUCTION}

When the loop diagrams which contribute to the electric dipole moments of quarks are evaluated in theories with $P$ and $T$ violating interactions, in general one finds a $P$ and $T$ violating quark-photon vertex function $\tilde{\Gamma}^{\mu_{q}}$ of the form

$$
\tilde{\Gamma}^{\mu_{q}}=\left[D_{1, q}\left(p_{i}+p_{f}\right)^{\mu} \gamma_{5}+\imath D_{2, q} \sigma^{\mu \nu}\left(p_{i}-p_{f}\right)_{\nu} \gamma_{5}\right] Q_{q}
$$

For convenience, we have extracted a factor $Q_{q}$, the charge of the quark, in units of the positron charge. Heretofore it has been the standard practice to use the $\gamma_{5}$ form of the Gordon identity, valid on mass shell,

$$
\bar{u}\left(p_{f}\right)\left[\left(p_{i}+p_{f}\right)^{\mu} \gamma_{5}\right] u\left(p_{i}\right)=\bar{u}\left(p_{f}\right)\left[\imath \sigma^{\mu \nu} q_{\nu} \gamma_{5}\right] u\left(p_{i}\right)
$$

with

$$
q^{\mu}=p_{i}^{\mu}-p_{f}^{\mu}
$$

to write the quark electric dipole moment interaction in its on mass shell form

$$
\tilde{\Gamma}^{\mu_{q, o m s}}=\imath D_{q} Q_{q} \sigma_{\mu \nu}\left(p_{i}-p_{f}\right)^{\nu} \gamma_{5}
$$

and to identify $d_{q}=D_{q} Q_{q}=\left[D_{1, q}+D_{2, q}\right] Q_{q}$ as the electric dipole moment of the quark. Note that the dimension of $d_{q}$ is $M^{-1}$, and instead of introducing $d_{q}$ one could have made the natural units explicit and introduced a gyroelectric ratio $h_{q}$, related to $d_{q}$ by $d_{q}=$ $h_{q} Q_{q} e /\left(2 m_{q}\right)$. However, unlike the practice in dealing with magnetic moments, it is standard practice to use $d_{q}$ rather than $h_{q}$. For further convenience we also write $d_{i, q}=D_{i, q} Q_{q}$.

In a previous paper [目], the quark contribution to the electric and magnetic dipole moments of the rho meson were calculated using vertices and propagators obtained from studies of the Dyson-Schwinger and Bethe-Salpeter equations. This provided some insight into the effect that QCD and confinement had on the electromagnetic properties of hadrons. The electric dipole moment of the quark used in that calculation was the on mass shell form of Eq. (雨). 
It should be noted that that the T-violating $N N \gamma$ vertex of equation (四) is gauge invariant only when $p_{i}^{2}=p_{f}^{2}$. However, this is enough for us to obtain a gauge invariant result for the $P$ and $T$ violating $\rho-\gamma$ coupling. As an off shell amplitude, it need not be gauge invariant - but it must satisfy the Ward Takahashi identities. We will examine the consequences of this requirement below.

In this paper we extend that study to examine the effects of the vertex function ambiguity off shell, and calculate the electric dipole moment of the rho meson which is generated by the $P$ and $T$ odd vertex function $\tilde{\Gamma}_{q}^{\mu}$ of Eq. (11). We continue to use the semi-phenomenological $n$ point functions of Quantum Chromodynamics obtained in the Dyson-Schwinger and BetheSalpeter framework. For ease of comparison with previous results we also give perturbative, bag model and non-relativistic results, as in our previous paper [1]. The methodology employed here allows us to investigate to what extent, when quarks are confined within a hadron, the two terms give equivalent results.

We emphasize that the rho electric dipole moment is studied as a model problem. The hadron electric dipole moment which has been extensively studied experimentally and theoretically is the neutron electric dipole moment. The relevant calculations are reviewed in [2]. We suggest that our results for the rho meson give an indication of the effects that one may expect for other hadron systems, including the neutron, when one has available techniques for exploring the off mass shell behaviour of the wavefunctions].

For a review of the topic of Dyson-Schwinger equations and their application to hadron phenomenology see [3]. For examples of the use of the Dyson-Schwinger and Bethe-Salpeter equations in the calculation of hadronic properties see references within [1, 3].

As in our previous paper, use will be made of forms for the quark propagator which ensure confinement by having no Lehmann representation, i.e. the quarks cannot go on mass-shell. Three different phenomenological forms for the rho amplitude will again be

\footnotetext{
${ }^{1} \mathrm{~A}$ start was made on that investigation by Costella [县]
} 
used. In that paper we used a dressed quark-photon vertex to ensure that both the WardTakahashi Identity and multiplicative renormalizability are preserved. In our present work we concentrate on the contribution of the $P$ and $T$ violating vertex function of Eq. (何), which we do not dress.

\section{THE INGREDIENTS OF THE CALCULATION}

$\mathrm{y}$

\section{A. The $\rho \rho \gamma$ Vertex}

The form for the $\rho \rho \gamma$ vertex is given by [1.5]

$$
\begin{aligned}
V_{\alpha \mu \beta}= & d_{\alpha}^{\nu}(p)\left[P_{\mu}\left(-g_{\nu \sigma} \mathcal{E}\left(q^{2}\right)+q_{\nu} q_{\sigma} \mathcal{Q}\left(q^{2}\right)\right)\right. \\
& \left.+\left(g_{\mu \nu} q_{\sigma}-g_{\mu \sigma} q_{\nu}\right) \mathcal{M}\left(q^{2}\right)+\varepsilon_{\nu \sigma \mu \rho} q^{\rho} \mathcal{D}\left(q^{2}\right)\right] d_{\beta}^{\sigma}\left(p^{\prime}\right)
\end{aligned}
$$

with $p$ the initial momentum of the rho, $p^{\prime}$ the final momentum and $q$ the momentum of the photon. The $d_{\alpha \beta}$ are the rho spin projection operators

$$
d_{\alpha \beta}(k)=g_{\alpha \beta}-\frac{k_{\alpha} k_{\beta}}{k^{2}}
$$

The form factors $\mathcal{E}, \mathcal{Q}, \mathcal{M}$ and $\mathcal{D}$ are interpreted in the limit as $q^{2} \rightarrow 0$ through $e \mathcal{E}(0)=e$, the charge of the rho, $e \mathcal{M}(0) /(2 m)=\mu$, the magnetic moment, $\mathcal{Q}(0)$ is related linearly to $Q$, the quadrupole moment, and $e \mathcal{D}(0) /(2 m)=d$, the electric dipole moment of the rho.f The integral for the rho EDM is too difficult to perform analytically. This means that to isolate the relevant terms they will be projected out of the integral before it is integrated numerically. Using a projection operator given by

\footnotetext{
${ }^{2}$ We use $m$ without subscripts for the mass of the $\rho^{+}$. All other masses in this paper are identified with the appropriate particle by subscripts.
} 


$$
P^{\alpha \mu \beta}=-\frac{1}{2 q^{2}} d^{\alpha \nu^{\prime}}(p) \varepsilon_{\nu^{\prime} \sigma^{\prime}}^{\mu \rho} d^{\beta \sigma^{\prime}}\left(p^{\prime}\right)
$$

the following result holds

$$
P^{\alpha \mu \beta} V_{\alpha \mu \beta} \stackrel{q \rightarrow 0}{\longrightarrow} \mathcal{D}(0)
$$

\section{B. Quark Contribution to the Electric Dipole Moment}

All calculations below are carried out in Euclidean space with co-ordinates $x_{1}, x_{2}, x_{3}, x_{4}$, and metric and gamma matrices given by $g_{\mu \nu}=\delta_{\mu \nu}, \gamma_{\mu}=\gamma_{\mu}^{\dagger}$ and $\left\{\gamma_{\mu}, \gamma_{\nu}\right\}=2 \delta_{\mu \nu}$. Treating the $u$ and $d$-quarks as identical except for their charge, the impulse approximation to the quark contribution to the electric moment is given by

$$
\begin{aligned}
\tilde{I}_{\alpha \mu \beta}= & (-1) \int \frac{d^{4} k}{(2 \pi)^{4}} \operatorname{tr}_{C F D}\left[\bar{\Gamma}_{\beta}^{\rho}(k ; p-q) S\left(k_{-+}\right) \imath \tilde{\Gamma}_{\mu, q}\left(k_{++}, k_{-+}\right)\right. \\
& \left.\times S\left(k_{++}\right) \Gamma_{\alpha}^{\rho}(k+q / 2 ; p) S\left(k_{+-}\right)\right] .
\end{aligned}
$$

where $k_{\alpha \beta}=k+\frac{\alpha q}{2}+\frac{\beta p}{2}, \Gamma_{\alpha}^{\rho}$ refers to the rho meson amplitude and $\bar{\Gamma}_{\alpha}^{\rho}(k ; p)=C^{\dagger} \Gamma_{\alpha}^{\rho}(-k ; p) C$ with $C$ the charge conjugation operator, $\gamma_{2} \gamma_{4}$. $\tilde{\Gamma}_{\mu, q}$ is the $P$ and $T$ violating quark-photon vertex and $S(p)$ is the dressed quark propagator for a quark of momentum $p$, all of which will be discussed below. The $\operatorname{tr}_{C F D}$ operation is a trace over colour, flavour and Dirac indices. A tilde has been placed on $I_{\alpha \mu \beta}$ to emphasize that this integral gives only the $P$ and $T$ violating rho-photon vertex.

\section{Quark Propagators}

The general form for the solution to the quark propagator Dyson-Schwinger equation [3] is

$$
\begin{aligned}
S(p) & =-\imath \not p \sigma_{V}\left(p^{2}\right)+\sigma_{S}\left(p^{2}\right) \\
& =\left(\imath \not p A\left(p^{2}\right)+B\left(p^{2}\right)\right)^{-1} .
\end{aligned}
$$


A model form for the propagator is given by [6, []

$$
\begin{aligned}
\bar{\sigma}_{S}(x)= & C_{\bar{m}} e^{-2 x}+\left(\frac{1-e^{-b_{1} x}}{b_{1} x}\right)\left(\frac{1-e^{-b_{3} x}}{b_{3} x}\right)\left(b_{0}+b_{2}\left(\frac{1-e^{-\Lambda x}}{\Lambda x}\right)\right) \\
& +\frac{\bar{m}}{x+\bar{m}^{2}}\left(1-e^{-2\left(x+\bar{m}^{2}\right)}\right), \\
\bar{\sigma}_{V}(x)= & \frac{2\left(x+\bar{m}^{2}\right)-1+e^{-2\left(x+\bar{m}^{2}\right)}}{2\left(x+\bar{m}^{2}\right)^{2}}-\bar{m} C_{\bar{m}} e^{-2 x},
\end{aligned}
$$

where $x=p^{2} / 2 D, \bar{\sigma}_{V}(x)=(2 D) \sigma_{V}\left(p^{2}\right), \bar{\sigma}_{S}=\sqrt{2 D} \sigma_{S}\left(p^{2}\right)$ and $\bar{m}=m / \sqrt{2 D}, D$ is a mass scale. $\left(\Lambda=10^{-4}\right.$ is chosen to decouple the small and large spacelike $p^{2}$ behaviour in Eq. (11); i.e. to allow $b_{0}$ to govern the ultraviolet behaviour and $b_{2}$ the infrared.) The parameters $C_{\bar{m}}, \bar{m}, b_{0}, \ldots, b_{3}$ are [B], $C_{\bar{m} \neq 0}=0.0, C_{\bar{m}=0}=0.121, \bar{m}=0.00897, b_{0}=0.131, b_{1}=2.90$, $b_{2}=0.603$ and $b_{3}=0.185$, with the mass scale $D=0.160 \mathrm{GeV}^{2}$ chosen to give the correct value for $f_{\pi}$. Note that with these values of the parameters the mean light quark mass is $5.0 \mathrm{MeV}$. This form for the quark propagator is based upon studies of the Dyson-Schwinger equation for $S(p)$ using a gluon propagator with an infrared singularity,

$$
g^{2} D_{\mu \nu}(k) \equiv\left(\delta_{\mu \nu}-\frac{k_{\mu} k_{\nu}}{k^{2}}\right) 8 \pi^{4} D \delta^{4}(k),
$$

and a dressed quark-gluon vertex [0,8]. A sufficient condition for the lack of free quark production thresholds is the absence of timelike poles in the propagator. The model quark propagator given above is an entire function (except at timelike $p^{2}=-\infty$ ) and so does not have a Lehmann representation. This means it can be interpreted as describing a confined particle and it ensures the lack of the unphysical singularities corresponding to free quarks in $\tilde{I}_{\alpha \mu \beta}$.

The electric dipole moment is also calculated using a form for the quark propagator developed by Mitchell and Tandy [9], to investigate $\rho-\omega$ mixing. This propagator is given by

$$
\begin{aligned}
& \bar{\sigma}_{S}(x)=C_{\bar{m}} e^{-2 x}+\frac{\bar{m}}{x}\left(1-e^{-2 x}\right), \\
& \bar{\sigma}_{V}(x)=\frac{e^{-2 x}-(1-2 x)}{2 x^{2}}-\bar{m} C_{\bar{m}} e^{-2 x} .
\end{aligned}
$$


To fix the parameters $\lambda=\sqrt{2 D}$ and $C_{\bar{m}}$, a fit to $\langle\bar{q} q\rangle, f_{\pi}, r_{\pi}$ and the $\pi$ - $\pi$ scattering lengths was done. With $\frac{1}{2}\left(m_{u}+m_{d}\right)=16 \mathrm{MeV}$ a best fit was obtained for $\lambda=0.889 \mathrm{GeV}$ and $C_{\bar{m}}=0.581$ [9]. This form has a deficiency which can be seen in its failure to correctly model the behaviour of $\sigma_{S}$ away from $x=0$, in the massless limit with dynamically broken chiral symmetry [0]. The large value for the mass of the quark used is related to this deficiency of the propagator [10].

\section{Rho Meson Amplitude}

The dominant Bethe-Salpeter amplitude for the rho meson is given by [1].[12],

$$
\Gamma_{\rho \mu}^{l}(k, p)=\imath\left(\gamma_{\mu}-\frac{\not p p_{\mu}}{p^{2}}\right) \tau^{l} \frac{\Gamma_{\rho}(k, p)}{N_{\rho}},
$$

where $k$ is the relative momentum of the quark and anti-quark, $p$ is the momentum of the rho meson and $l$ and $\mu$ are flavour and Dirac indices respectively. This form ignores other allowable Dirac structure in the vector meson Bethe-Salpeter amplitude and so introduces errors of the order of $10 \%$ [13]. Using the quark propagator defined in Eq. (111) and a Ball-Chiu quark-photon vertex (see later), Chappell uses the following approximate form for $\Gamma_{\rho}(k, p)$ 14

$$
\Gamma_{\rho}=e^{-k^{2} / a_{1}^{2}}+\frac{a_{2}}{1+\frac{k^{2}}{\alpha a_{1}}},
$$

with $a_{1}=0.38845 \mathrm{GeV}, a_{2}=0.01478, \alpha=2 \mathrm{GeV}$. The values for the parameters were found by fitting to the experimental values of $f_{\rho}$ and $g_{\rho \pi \pi}$ [14].

Pichowsky and Lee, also using the quark propagator defined in Eq. (11) and a quarkphoton vertex of the Ball-Chiu type, use an identical form for $\Gamma_{\rho}$, given by [15]

$$
\Gamma_{\rho}(k, p)=e^{-k^{2} / a_{V}^{2}}+\frac{c_{V}}{1+k^{2} / b_{V}^{2}},
$$

where $a_{V}=0.400 \mathrm{GeV}, b_{V}=0.008 \mathrm{GeV}$ and $c_{V}=125.0$. Note that the coefficient of the rational term in the vertex function, and the scale factor, differ dramatically in the two attempts to fit this form of vertex function, 
Mitchell and Tandy, with $S(p)$ defined by Eqs. (13 and 14), use a form for the amplitude $\Gamma_{\rho}$ given by

$$
\Gamma_{\rho}(k, p)=e^{-k^{2} / a^{2}}
$$

with $a=0.194 \mathrm{GeV}$. The momentum scale in the exponential has been reduced by a factor of 2 and the rational term in the vertex function has been omitted.

The normalisation for the rho amplitude is fixed by [15

$$
\begin{aligned}
p_{\mu}\left(\delta_{\alpha \beta}-\frac{p_{\alpha} p_{\beta}}{p^{2}}\right)= & N_{c} \operatorname{tr}_{D} \int \frac{d^{4} k}{(2 \pi)^{4}} \frac{\partial S\left(k_{+}\right)}{\partial p_{\mu}} \Gamma_{\rho \alpha}(k, p) S\left(k_{-}\right) \Gamma_{\rho \beta}(k, p) \\
& +N_{c} \operatorname{tr}_{D} \int \frac{d^{4} k}{(2 \pi)^{4}} S\left(k_{+}\right) \Gamma_{\rho \alpha}(k, p) \frac{\partial S\left(k_{-}\right)}{\partial p_{\mu}} \Gamma_{\rho \beta}(k, p)
\end{aligned}
$$

where $k_{\alpha}=k+\frac{\alpha p}{2}$. This condition, with the fact that the quark-photon vertex obeys the Ward Identity, ensures that $\mathcal{E}\left(q^{2}=0\right)=1$, i.e. that the rho has unit charge [7].

\section{E. The Quark-Photon Vertex}

The quark-photon vertex also satisfies its own Dyson- Schwinger equation, but solving this integral equation is difficult. Despite this, a realistic ansatz for the vertex function has been developed 16 19].

The quark-photon vertex ansatz thus obtained is given by

$$
\Gamma_{\mu}^{\mathrm{BC}+\mathrm{CP}}(p, q)=Q_{q}\left[\Gamma_{\mu}^{\mathrm{BC}}(p, q)+\Gamma_{\mu}^{\mathrm{CP}}(p, q)\right]
$$

The Ball-Chiu vertex, $\Gamma_{\mu}^{\mathrm{BC}}$ has the form [18]

$$
\begin{aligned}
\Gamma_{\mu}^{\mathrm{BC}}(p, q)= & \frac{A\left(p^{2}\right)+A\left(q^{2}\right)}{2} \gamma_{\mu}+\frac{(p+q)_{\mu}}{p^{2}-q^{2}}\left[\frac{1}{2}\left(A\left(p^{2}\right)-A\left(q^{2}\right)\right)(\not p+\not q)\right. \\
& \left.-\imath\left(B\left(p^{2}\right)-B\left(q^{2}\right)\right)\right]
\end{aligned}
$$

This vertex ansatz is completely described by the dressed quark propagator and satisfies both the Ward-Takahashi and Ward Identities, is free of kinematic singularities as $q^{2} \rightarrow p^{2}$, 
transforms correctly under appropriate transformations and reduces to the perturbative result in the appropriate limit.

To ensure multiplicative renormalizability Curtis and Pennington added a transverse piece to the Ball-Chiu vertex [19]. This term has the form

$$
\Gamma_{\mu}^{\mathrm{CP}}(p, q)=\left(\frac{-\imath \gamma_{\mu}\left(p^{2}-q^{2}\right)-(p+q)_{\mu}(\not p-\not q)}{2 d(p, q)}\right)\left(A\left(p^{2}\right)-A\left(q^{2}\right)\right)
$$

with

$$
\begin{gathered}
d(p, q)=\frac{1}{p^{2}+q^{2}}\left[\left(p^{2}-q^{2}\right)^{2}+\left(M^{2}\left(p^{2}\right)+M^{2}\left(q^{2}\right)\right)^{2}\right], \\
M\left(p^{2}\right)=\frac{B\left(p^{2}\right)}{A\left(p^{2}\right)} .
\end{gathered}
$$

We introduce the quark electric dipole moment through the vertex function $\tilde{\Gamma}^{\mu}$ of Eq. (1). We have not attempted to "dress" this vertex by including gluon loop corrections.

To have an explicit example in mind we note that in models where the quark electric dipole moment is generated by Higgs exchange [20,21], the structure of the quark electric dipole moment is that of Eq. (11). With the charged Higgs-quark interaction Lagrangian given by

$$
\mathcal{L}_{\text {int }}=2^{3 / 4} G_{F}^{1 / 2} \bar{U}\left[V_{K M} M_{D} \alpha H^{+} R+M_{U} V_{K M} \beta H^{+} L\right] D+H . C .
$$

the electric dipole moments of the up and down quarks are dominated by the bottom and the top quark loops respectively. With a charged Higgs mass of the order of the top mass, the electric dipole moment of the down quark is significantly larger than that of the up quark, and the $P$ and $T$ violating $d-\gamma$ vertex is given by Eq. (1), with

$$
\begin{aligned}
d_{1, d} & =K\left(F_{1}\left(m_{t}^{2} / M_{H}^{2}\right)+F_{2}\left(m_{t}^{2} / M_{H}^{2}\right)\right) \\
d_{2, d} & =-K F_{1}\left(m_{t}^{2} / M_{H}^{2}\right) \\
K & =\frac{G_{F} m_{d}}{48 \pi^{2}}\left|V_{d q}\right|^{2} \operatorname{Im}\left(\alpha^{*} \beta\right) \\
F_{1} & =4 \frac{x}{1-x}\left(1+\frac{x}{1-x} \ln x\right) \\
F_{2} & =\frac{x}{(1-x)^{2}}\left(5 x-3+\frac{2(3 x-2)}{1-x} \ln x\right),
\end{aligned}
$$


Note that, in the on mass shell approximation, the $F_{1}$ terms cancel, leaving only the $F_{2}$ terms.

\section{F. Gauge Invariance, Ward Identities, Renormalization and Off-Shell behaviour}

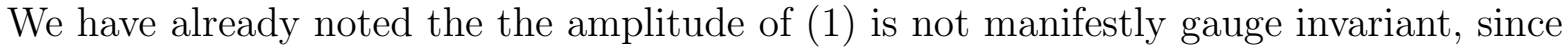

$$
\partial_{\mu} \tilde{\Gamma}_{q}^{\mu}=d_{1 q}\left(p_{i}^{2}-p_{f}^{2}\right) \gamma_{5}
$$

which vanishes only when $p_{i}^{2}=p_{f}^{2}$, and thus vanishes on mass shell. However, because $\tilde{\Gamma}_{q}^{\mu}$ is a Green's function, not an element of the $S$-matrix, it need only satisfy the Ward-Takahashi identity [22],

$$
\tilde{\Gamma}_{\mu}(p, p)=-\frac{\partial \Sigma_{5}(p)}{\partial p_{\mu}}
$$

or

$$
q_{\mu} \tilde{\Gamma}^{\mu}\left(p_{i}, p_{f}\right)=-\left(\Sigma_{5}\left(p_{i}\right)-\Sigma_{5}\left(p_{f}\right)\right)
$$

The $C P$-violating term, $\Sigma_{5}(p)$, in the quark self energy, required to satisfy the Ward Identity, will be generated by the same process that generates the $T$ violating vertex, for example by Higgs loops. In general

$$
\Sigma_{5}(p)=m_{5}(p) \gamma_{5}
$$

and $\Sigma_{5}(p)$ is divergent, coming from a one loop diagram. There are however no terms of this structure in the unrenormalised lagrangian, so we have no parameter to adjust in the renormalisation process. In $Q E D$ this is not a problem, as one can demand that the equivalent term vanish on mass shell [23]. In the present case quarks do not have a mass shell in the confined phase of $Q C D$, and the best we can do is to renormalize $\Sigma_{5}$ such that it vanishes at some renormalization point, $\mu$. That is, we have, after renormalisation,

$$
\Sigma_{5}(p)=-d_{1}\left(p^{2}-\mu^{2}\right) \gamma_{5}
$$


guaranteeing the Ward-Takahashi identities?. For non-confined particles the choice $\mu=m$, where $m$ is the mass of the particle concerned, recovers the results of [23.

To lowest order then, the corrections to the quark-photon vertex due to Higgs loops are given by the sum of diagrams given in Fig. (代. If $\mu$ is set equal to $m_{q}$ then the "nonstandard" quark-photon vertex term in Eq. (1) is cancelled, with the result that

$$
\tilde{\Gamma}_{q}^{\mu}=\left(d_{2}+d_{1}\right) \imath \sigma^{\mu \nu} q_{\nu} \gamma_{5}
$$

just as if we had used the on-shell identity of Eq. (2). If, on the other hand, one renormalizes $\Sigma_{5}$ at some other point $\mu \neq m_{q}$, then there is no such cancelation but $\tilde{\Gamma}_{q}^{\mu}$ has a $\mu^{2}$ dependence. This $\mu^{2}$ dependence is a result of the absence of an adequate theory of perturbations about the confined phase, indeed of an absence of an adequate theory of the confined phase. This being the case, to make progress we set set $\mu^{2}=\left\langle p_{a v}\right\rangle^{2}$, with the result that the last two diagrams of Fig. (11) give negligible contributions and so

$$
\tilde{\Gamma}_{q}^{\mu}=d_{1}\left(p_{i}+p_{f}\right)^{\mu} \gamma_{5}+d_{2} \imath \sigma^{\mu \nu} q_{\nu} \gamma_{5}
$$

just as Eq.(1).

It may be thought that the lack of gauge invariance of the $C P$ violating vertex of (囵) would lead to a lack of gauge invariance for the electric dipole $\rho \rho \gamma$ coupling. Explicit calculation shows this not to be the case - the resulting electric dipole $\rho \rho \gamma$ coupling has only the gauge invariant form $\varepsilon_{\nu \sigma \mu \rho} q^{\rho}$. While a calculation of the neutron EDM using the $T$ and $P$ violating vertex of Eq. (1) in a three quark model of the nucleon is at present impractical, if one uses it in a quark-diquark model of the nucleon, the resulting $N N \gamma, T$ and $P$ violating, vertex is similarly gauge invariant.

\footnotetext{
${ }^{3}$ At least when we can regard $d_{1}$ as independent of $p_{i}$ and $p_{f}$
} 


\section{THE DIPOLE MOMENT}

The dipole moment of the rho can now be calculated. The colour, flavour and Dirac traces are performed for the integral $\tilde{I}_{\alpha \mu \beta}$, see appendix A, and the dipole term projected out using the projection operator $P^{\alpha \mu \beta}$. The integral is then performed numerically using Gaussian quadrature methods to obtain the results given in Table —.

For comparison, we also use perturbation theory to calculate the electric dipole moment of a rho meson made of free, undressed quarks. To do this for the $\left(p_{i}+p_{f}\right)^{\mu} \gamma_{5}$ term integral $\tilde{I}_{\alpha \mu \beta}$, the following replacements are made,

$$
\begin{aligned}
S(p) & \rightarrow 1 /(\imath \not p+m) \\
\Gamma^{\rho}(k, p) & \rightarrow 1 \\
\tilde{\Gamma}_{\mu}\left(k_{++}, k_{-+}\right) & \rightarrow\left(k_{++}+k_{-+}\right)_{\mu} \gamma_{5} D_{1, q} Q_{q} .
\end{aligned}
$$

Using the standard dimensional regularization scheme along with the Feynman parameterization technique [24], the integral can be shown to remain finite. However, as was shown in our earlier paper [1], the corresponding contribution to the electric charge is infinite. Thus calculating the contribution to the rho dipole moment in perturbation theory via the ratio $\mathcal{D}(0) / \mathcal{E}(0)$ yields

$$
d_{\rho ; \text { pert }}=0
$$

In [1] the rho EDM was also calculated in the bag model. As it has been noted previously that $\bar{\psi}\left(p_{i}+p_{f}\right)_{\mu} \gamma_{5} \psi=\bar{\psi} \imath \sigma_{\mu \nu} q^{\nu} \gamma_{5} \psi$ for quarks on the mass-shell, it follows that the nonstandard form for the quark EDM will yield the same bag model result as the standard term, since the quarks satisfy the equation of motion inside the bag.

\footnotetext{
${ }^{4}$ the equivalent calculation for the $\imath \sigma^{\mu \nu} q_{\nu} \gamma_{5}$ contribution was performed in our earlier paper 迎, with the result that the rho electric dipole moment is $d_{\rho}=\left(m_{u} d_{u}+m_{d} d_{d}\right) /(2 m)$ in this perturbative limit.
} 
In the non-relativistic limit, the result is simply

$$
d_{\rho}^{(N R)}=\left\{\left(d_{1, u}+d_{2, u}\right)+\left(d_{1, d}+d_{2, d}\right)\right\}
$$

The results may be expressed in the form

$$
d_{\rho}=\left(d_{1, u}+d_{1, d}\right) A+\left(d_{2, u}+d_{2, d}\right) B
$$

For our various choices of input, $\mathrm{A}$ and $\mathrm{B}$ are given in Table $\mathbf{1}$.

\section{DISCUSSION}

Using a model form for the quark propagator obtained from the Dyson- Schwinger equations, two different phenomenological rho-meson amplitudes fitted to $f_{\rho}$ and $g_{\rho \pi \pi}$ and one used to describe $\rho-\omega$ mixing, and a quark-photon vertex which incorporated the $P$ and $T$ violating quark-photon vertex function of Eq. (四), we have calculated the electric dipole moment of the rho.

For each of the coefficients $A$ and $B$, all three non-perturbative models agree qualitatively with the Bethe-Salpeter amplitude of [15] yielding the largest value for $d_{\rho}$ and that of [9] the smallest. The results for the amplitudes of Chappell, and Pichowsky and Lee, are very close, which is indicative of the similarities of the two approaches.

For the same non-perturbative model, a comparison of the coefficients $A$ and $B$ shows that the contribution of the $\left(p_{i}+p_{f}\right)_{\mu} \gamma_{5}$ term is rather less than that of the "standard" $\imath \sigma_{\mu \nu} q^{\nu} \gamma_{5}$ term. The $\left(p_{i}+p_{f}\right)_{\mu} \gamma_{5}$ contribution was $\sim 67 \%$ of the $\imath \sigma_{\mu \nu} q^{\nu} \gamma_{5}$ result for the amplitude developed by Mitchell and Tandy, 83\% for that of Chappell and $~ 81 \%$ for the amplitude of Pichowsky and Lee, with the $\left(p_{i}+p_{f}\right)_{\mu} \gamma_{5}$ and $\imath \sigma_{\mu \nu} q^{\nu} \gamma_{5}$ terms weighted equally.

In the Bag Model, and in the non-relativistic limit, these two terms contribute equally. In the perturbative, free limit, on the other hand the $\sigma_{\mu \nu} q^{\nu} \gamma_{5}$ term gives a finite (but small) contribution, whereas the $\left(p_{i}+p_{f}\right)_{\mu} \gamma_{5}$ term gives a vanishing contribution. In a sense the non-perturbative models interpolate between these limits. 
To be explicit, consider the Higgs model introduced above. We take the Higgs mass to be $100 \mathrm{GeV}$, and the top mass to be $170 \mathrm{GeV}$, and note that, in this model, $d_{d} \gg d_{u}$. Eqs. (25)-(29) show that the $\left(p_{i}+p_{f}\right)^{\mu} \gamma_{5}$ term has a coefficient more than twice as large as that of the standard term, i.e. $d_{1, d} / d_{2, d}=2.34$. The resulting rho electric dipole moment, expressed as a fraction of the non-relativistic value is given in Table [1].

In this case the models of Chappell, and Pichowsky and Lee, give similar results which are about two thirds of the non-relativistic result. The Mitchell and Tandy result is significantly smaller. The good news is that the standard non-relativistic result is good to a factor of 2 to 3 , and that while one is concerned with approximate estimates it is a reasonable guide to the overall effect.

Though this calculation was carried out for the rho meson it leads to some interesting speculation about the calculation of the quark contribution to the neutron electric dipole moment, which is of interest in the study of CP-violation. The use of the full off mass shell $P$ and $T$ violating interaction of Eq. (11) could significantly alter both the standard model and the many non-standard model results for the neutron EDM. While one can expect the order of magnitude of the existing calculations to be correct, at a precision level the off-mass shell effects considered here will become important. As some of the non-standard model calculations yield values that are close to the current experimental limits [2], it will become important to attempt precision calculations in the future. Of course, when such calculations are attempted, many additional sources of a neutron electric dipole moment will need to be considered. In the Higgs model, these include

1. charged Higgs contributions to

(a) the quark colour-electric dipole moment

(b) the gluon colour-electric dipole moment

(c) other $P$ and $T$ odd quark-quark interactions

2. neutral Higgs contributions to the above 


\section{3. hadronic effects}

Considerations similar to those discussed here will enter the calculation of the quark colourelectric dipole moment effects, and possibly also the other additional effects.

It is well known that the fact that the quarks in hadrons are both relativistic and bound, and thus are significantly off their mass shell, has significant effects on the quark contribution to the magnetic moments of hadrons [1,25]. The study of the rho meson presented here shows that similar effects occur in the calculation of the electric dipole moments of hadrons. In particular, it is necessary to differentiate between the two forms of the $P$ and $T$ odd quark-photon interaction in these calculations, and not to treat them as being equivalent as they are on the quark mass shell. The next generation of calculations of the electric dipole moment of the neutron should take such effects into account.

\section{APPENDIX A: INTEGRAL FOR THE ELECTRIC DIPOLE MOMENT}

Taking the colour and flavour traces, the integral becomes

$$
\begin{aligned}
\tilde{I}_{\alpha \mu \beta}= & \frac{2 \imath N_{c}}{N_{\rho}^{2}} \int \frac{d^{4} k}{(2 \pi)^{4}} \Gamma_{\rho}(k) \Gamma_{\rho}(k+q / 2) t r_{D}[ \\
& +\gamma_{\beta} \not k_{-+} \gamma_{5} \not k_{++} \gamma_{\alpha} \tilde{k}_{\mu} T_{1}+\gamma_{\beta} \not k_{-+} \gamma_{5} \gamma_{\alpha} \not k_{+-} \tilde{k}_{\mu} T_{2} \\
& +\gamma_{\beta} \not k_{-+} \gamma_{5} \sigma_{\mu \rho} q^{\rho} \not k_{++} \gamma_{\alpha} T_{3}+\gamma_{\beta} \not k_{-+} \gamma_{5} \sigma_{\mu \rho} q^{\rho} \gamma_{\alpha} \not k_{+-} T_{4} \\
& +\gamma_{\beta} \gamma_{5} \not k_{++} \gamma_{\alpha} \not k_{+-} \tilde{k}_{\mu} T_{5} \\
& \left.+\gamma_{\beta} \gamma_{5} \sigma_{\mu \rho} q^{\rho} \not k_{++} \gamma_{\alpha} \not k_{+-} T_{6}+\gamma_{\beta} \gamma_{5} \sigma_{\mu \rho} q^{\rho} \gamma_{\alpha} T_{7}\right],
\end{aligned}
$$

where $\tilde{k}_{\mu}=\left(k_{++}+k_{-+}\right)_{\mu}$, only $P$ and $T$ odd terms have been retained, and terms which will not survive the Dirac trace have been omitted. The $T_{1-7}$ are defined by

$$
\begin{aligned}
& T_{1}=-V_{1} \sigma_{V}\left(k_{-+}^{2}\right) \sigma_{V}\left(k_{++}^{2}\right) \sigma_{S}\left(k_{+-}^{2}\right), \\
& T_{2}=-V_{1} \sigma_{V}\left(k_{-+}^{2}\right) \sigma_{S}\left(k_{++}^{2}\right) \sigma_{V}\left(k_{+-}^{2}\right), \\
& T_{3}=-V_{2} \sigma_{V}\left(k_{-+}^{2}\right) \sigma_{V}\left(k_{++}^{2}\right) \sigma_{S}\left(k_{+-}^{2}\right), \\
& T_{4}=-V_{2} \sigma_{V}\left(k_{-+}^{2}\right) \sigma_{S}\left(k_{++}^{2}\right) \sigma_{V}\left(k_{+-}^{2}\right),
\end{aligned}
$$




$$
\begin{aligned}
& T_{5}=-V_{1} \sigma_{S}\left(k_{-+}^{2}\right) \sigma_{V}\left(k_{++}^{2}\right) \sigma_{V}\left(k_{+-}^{2}\right), \\
& T_{6}=-V_{2} \sigma_{S}\left(k_{-+}^{2}\right) \sigma_{V}\left(k_{++}^{2}\right) \sigma_{V}\left(k_{+-}^{2}\right), \\
& T_{7}=V_{2} \sigma_{S}\left(k_{-+}^{2}\right) \sigma_{S}\left(k_{++}^{2}\right) \sigma_{S}\left(k_{+-}^{2}\right),
\end{aligned}
$$

with,

$$
\begin{aligned}
& V_{1}=-\left(d_{1, u}+d_{1, d}\right), \\
& V_{2}=-\imath\left(d_{2, u}+d_{2, d}\right) .
\end{aligned}
$$




\section{REFERENCES}

[1] M.B. Hecht and B.H.J. McKellar, Phys. Rev. C C57 2638 (1998).

[2] X-G. He, B.H.J. McKellar, and S. Pakvasa, Int. J. Mod. Phys. A 45011 (1989).

[3] C.D. Roberts and A.G. Williams, Prog. Part. Nucl. Phys 3324 (1994).

[4] J. P. Costella, B.Sc.(Hons.) Thesis "The Neutron Electric Dipole Moment in a Relativistic Constituent Quark Model", University of Melbourne (1990).

[5] A. Salam and R. Delbourgo, Phys. Rev. 1351398 (1964).

[6] C.D. Roberts, in "Chiral Dynamics: Theory and Experiment", Bernstein, A.M. and Holstein, B.R. (Eds.), Lecture Notes in Physics, Vol. 452, p. 68 (Springer, Berlin 1995).

[7] C.D. Roberts, Nucl. Phys A 605475 (1996).

[8] C.J. Burden, C.D. Roberts and M.J. Thomson, Phys. Lett. B 371163 (1996).

[9] K.L. Mitchell, P.C. Tandy, Phys. Rev. C 551477 (1997).

[10] K.L. Mitchell, Private Communications (1997).

[11] J. Praschifka, R.T. Cahill and C.D. Roberts, Int. J. Mod. Phys. A 44929 (1989).

[12] P. Jain and H. Munczek, Phys. Rev. D 485403 (1993).

[13] M.R. Frank and C.D. Roberts, Phys. Rev. C 53390 (1996).

[14] I. Chappell, Private Communications (1996).

[15] M.A. Pichowsky and T.-S.H. Lee, Phys. Rev. D 561644 (1997).

[16] C.J. Burden, C.D. Roberts and A.G. Williams, Phys. Lett. B 285347 (1992).

[17] Z. Dong, H.J. Munczek and C.D. Roberts, Phys. Lett. B 333536 (1994).

[18] J.S. Ball and T.W. Chiu, Phys. Rev. D 222542 (1980). 
[19] D.C. Curtis and M.R. Pennington, Phys. Rev. D 424165 (1990).

[20] T. D. Lee, Phys. Rev. D 8, 1226 (1973); Phys. Rep. 96, 143 (1974).

[21] S. Weinberg, Phys. Rev. Lett. 37, 657 (1976).

[22] C. Itzykson and J. Zuber, "Quantum Field Theory", McGraw-Hill, New York, 1980.

[23] G. Feinberg, P. Kabir, and S. Weinberg, Phys. Rev. Lett. 3, 527 (1959).

[24] F. Mandl and G. Shaw, "Quantum Field theory", John Wiley \& Sons, New York 1993.

[25] N.E. Tupper, B.H.J. McKellar, and R. Warner, Australian Journal of Physics 4119 (1988). 
TABLE I. Expansion coefficients $A$ and $B$ for the Electric Dipole Moment $d_{\rho}$

\begin{tabular}{|c|r|r|}
\hline \hline Model & $\mathrm{A}$ & $\mathrm{B}$ \\
\hline Chappell & 0.620 & 0.743 \\
Pichowsky and Lee & 0.627 & 0.779 \\
Mitchell \& Tandy & 0.418 & 0.627 \\
Perturbative & 0.000 & 0.828 \\
Bag Model & 1.000 & 0.010 \\
Non-Relativistic & & 1.000 \\
\hline \hline
\end{tabular}


TABLE II. Electric Dipole Moment $d_{\rho}$ in a Higgs model, as a ratio to the Non-Relativistic result

\begin{tabular}{|c|r|}
\hline \hline Model & Ratio \\
\hline Chappell & \\
Pichowsky and Lee & 0.657 \\
Mitchell \& Tandy & 0.672 \\
Perturbative & 0.465 \\
Bag Model & 0.003 \\
Non-Relativistic & 0.828 \\
\hline \hline
\end{tabular}




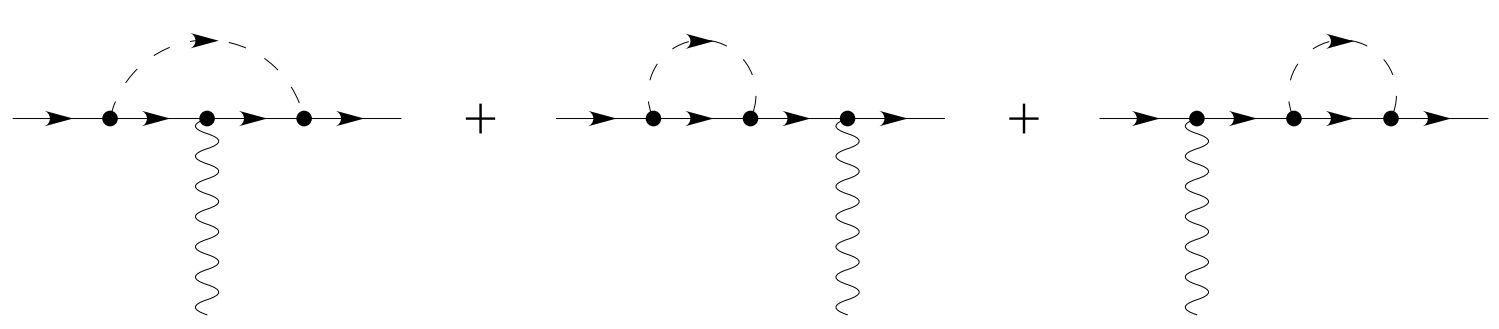

FIG. 1. Lowest order Higgs loop corrections to the quark-photon vertex 